\title{
Histochemical Detection of the Position of Metal Microelectrode
}

The detection of the precise position of the microelectrode in the tissue which is being examined is of vital importance in the analysis of electrophysiological processes on the spot examined, as well as in electrical stimulation of the determined biological structure, i.e. in working with both derivating and stimulating electrodes.

Our work covering the electrolymphocardiogram (ELCG) of the sacral lymph frog's hearts (Rana esculenta) by means of the metal microelectrode has confirmed this, and it prompted us in the first place to determine precisely the position of the electrodes. In order to accomplish this, we introduced iron from the microelectrode into the tissue by electrolysis, and subsequently determined the presence of iron in the tissue by histochemical methods in serial sections of the tissue.

Metal microelectrodes (diameter about $5 \mu$ ) were made of stainless steel wire (36 SWG Allen and Hanburys Ltd.) diameter $0.3 \mathrm{~mm}$, according to Bures et al. 1 .

The frog was anaesthetized with urethane and the skin from the sacral lymph hearts removed. Using the stereomicroscope, the microelectrode was inserted at the spot which showed the most intensive pulsations.

After registering ELCG the electrolysis was carried out by D.C. of $0.5 \mathrm{~V}$ intensity during $15 \mathrm{sec}$. The positive pole was connected with microelectrode and the negative pole with indifferent electrode inserted in the lower limb on the opposite side. The lymph heart was excised and native frozen sections were made and the histochemical reaction on iron (Fe II) performed ${ }^{2}$.
This method gave the positive reaction to iron (dark blue colour) in the examined tissue within a radius of $90 \mu$ from where the microelectrode had been situated.

Further work is in progress.

Zusammenfassung. Die Methode der histochemischen Detektion der Lage der Metallmikroelektrode wird beschrieben. Nachdem die Aktionpotentiale registriert worden sind, wird das Eisen aus der Mikroelektrode durch Elektrolyse ins Gewebe eingeführt, und anschliessend in Serienschnitten mit histochemischen Methoden nachgewiesen.

S. Gamulin, Lj. Gurović and P. NARANSCIK

Institute of Pathophysiology, Medical faculty, University of Zagreb (Yugoslavia). 12 August 1967.

1 L. BUREŠ, M. Petran and J. Z ZACHAR, in Electrophysiological Methods in Biological Research (Czechoslovak Academy of Sciences, Prague 1962), p. 160.

2 V. GADIK und V. Totović, in Biochemisches Taschenbuch (Ed. H. M. RaUEN; Springer, Berlin 1964), p. 486.

\section{Mesures en continu et in situ des pH artériel et veineux profonds chez le Mammifère}

La détermination du $\mathrm{pH}$ sanguin doit de toute nécessité être faite sans prélèvement de sang, de façon non-différee, au lieu même où l'on désire connaître la valeur du paramètre physico-chimique en question, en continu et de façon reproductible. Pour résoudre ce problème, qui vise à permettre l'établissement d'une Physiologie physiologique et hautement quantitative ${ }^{\mathfrak{x}}$, il convient de miniaturiser l'ensemble électrode de verre - électrode de référence de façon à rendre le capteur cathétérisable.

L'instrument que nous avons réalisé et que représentent les Figures 1 et 2 répond aux différentes préoccupations ci-dessus énoncées et il est caractérisé notamment par sa bonne sensibilité et sa parfaite rectilinéarité (Figure 3). La description détaillée de la construction de cette sonde est donnée ailleurs ${ }^{2}$ et nous ne signalerons ici que les points suivants: (a) l'électrode de $\mathrm{pH}$ est en verre "Corning 015 » de $2,5-3,2 \mathrm{~mm}$ de $\varnothing$ et de $50-100 \mu$ d'épaisseur pour la boule, sa résistance interne (Ri) va de $30-800 \mathrm{M} \Omega$ et sa sensibilité est d'autant plus grande que cette résistance est élevée. Les conditions d'obtention d'une bonne sensibilité sont: $1^{\circ}$ ) de tremper la boule après fabrication d'abord pendant une quinzaine d'heures au moins dans une solution $\mathrm{HCl} N / \mathrm{l}$ à la température du laboratoire, ensuite dans une solution $\mathrm{NaCl} M / 1,2^{\circ}$ ) d'éviter tout courtcircuit dont la cause principale est l'ascension capillaire du liquide de conservation ou à mesurer le long de la paroi extérieure du cylindre de verre "Corning" très hygroscopique ${ }^{3}$; (b) l'électrode de référence qui, dans nos premières réalisations, était en calomel est désormais une électrode dérivant de celle proposée par KENNARD ${ }^{4}$ et
Sonde à $\mathrm{pH}$

Fil blindé de liaison

Partie brasée

Couche de "Chicoprène"

Tube de polyéthylène de $\varnothing 5 \mathrm{~mm}$

Parties brasées

Couche de *Chicoprène "

Tube de polyéthylène de $\varnothing 1 \mathrm{~mm}$

Tube de polvéthylène protecteur

("Carter" du fil d'argent)

Cire à cacheter

Fil d'argent chloruré

Fil d'argent chloruré

Solution aqueuse de $\mathrm{HCl}$ à $0,1 \mathrm{~N}$

Solution aqueuse de $\mathrm{NaCl}$ à $0,1 \mathrm{~N}$

Partie brasée

Joint (facultatif) en "Beckacite

Electrode de référence, tube capillaire en verre "Pyrex"

Boule de verre "Corning 015 " de $\varnothing 2,5 \mathrm{~mm}$

Fig. 1

Echelle:4 